\title{
The Pair Correlation Function in Liquid ${ }^{4} \mathrm{He}$
}

\author{
Raymond D. Mountain and Harold J. Raveché \\ Institute for Basic Standards, National Bureau of Standards, Washington, D.C. 20234
}

(June 28, 1973)

\begin{abstract}
The pair correlation function obtained from the neutron diffraction data of Mozer, De Graaf, and Le Neindre, is given for liquid ${ }^{4} \mathrm{He}$ at several thermodynamic states above and below the superfluid transition. A method for smoothly and accurately extrapolating the pair function into the limit of zero internuclear separation is considered. The pair function is computed from an approximation integral equation for the ground state wave function and compared to the experimental results.
\end{abstract}

Key words: Ground state wave function; liquid ${ }^{4} \mathrm{He}$; neutron diffraction data; pair correlation function; short range behavior; structure factor; superfluid transition.

\section{Introduction}

The intensity of elastically scattered neutrons in an isotopic fluid defines $[1]^{1}$ the structure factor, $S(\kappa ; \rho, T)$, which is a function of $\kappa=\left|\boldsymbol{\kappa}-\boldsymbol{\kappa}_{0}\right|$, where $\boldsymbol{\kappa}_{0}$ is the wave vector of the neutron before scattering, and $\kappa$ the wave vector after scattering. The quantities $\rho$ and $T$ are respectively the density and temperature of the fluid sample. The structure factor is of particular interest as its Fourier transform, $g_{2}\left(r_{12} ; \rho, T\right)$, with respect to the internuclear distance $r_{12}$, defines the local structure of the fluid.

$g_{2}\left(r_{12} ; \rho, T\right)=1$

$+\left(2 \pi^{2} \rho r_{12}\right)^{-1} \int_{0}^{\infty} d \kappa \kappa \sin \kappa r_{12}[S(\kappa ; \rho, T)-1]$.

The function $g_{2}\left(r_{12} ; \rho, T\right)$ is necessarily positive and is usually referred to as the pair correlation function; we will adopt this nomenclature. Both neutron and x-ray [2] measurements of $S(\kappa ; \rho, T)$ in ${ }^{4} \mathrm{He}$ have been made, but data of high statistical accuracy, for several thermodynamic states in $1-3 \mathrm{~K}$ range, have only recently been reported [3]. The motivation for experiments at low temperatures is to explore the changes in the behavior of the local structure of liquid ${ }^{4} \mathrm{He}$ that might accompany the transition at $T=T_{\lambda}$ from normal to superfluid [4]. In addition, it is also known [5] that measurements of the isothermal density derivative of $g_{2}\left(r_{12} ; \rho, T\right)$ are a measure of the magnitude and range of the correlation between three atoms. Quite recently, Mozer, De Graaf, and Le Neindre

${ }^{1}$ Figures in brackets indicate the literature references at the end of this paper.
$[6,7]$ have done neutron diffraction measurements of high statistical accuracy for several thermodynamic states in liquid ${ }^{4} \mathrm{He}$. The thermodynamic states at which the scattering experiments were performed are listed in table 1. The table also includes the value of the superfluid transition temperature, $T_{\lambda}$, for each density considered. All temperatures in table 1 are known to within $\pm 0.005 \mathrm{~K}$, and all densities to within \pm 0.25 percent.

TABLE 1. Thermodynamic states for $\mathrm{g}_{2}(\mathrm{r} ; \rho, \mathrm{T})$ in liquid ${ }^{4} \mathrm{He}$ The bracketed number in the pressure column is the observed pressure in the Pascal unit; $1 \mathrm{~atm}=0.101325 \mathrm{MPa}$.

\begin{tabular}{c|c|c|c|c}
\hline \hline$\rho\left(\right.$ atoms $\left./ \AA^{3}\right)$ & $T(\mathrm{~K})$ & $T_{\lambda}(\mathrm{K})$ & $P($ atm $)$, & $(\mathrm{MPa})$ \\
\hline 0.0230 & 1.860 & 2.135 & 4.31 & $(0.44)$ \\
.0230 & 2.050 & 2.135 & 3.96 & $(0.40)$ \\
.0230 & 2.130 & 2.135 & 3.60 & $(0.37)$ \\
.0230 & 2.140 & 2.135 & 3.60 & $(0.37)$ \\
.0230 & 2.300 & 2.135 & 3.79 & $(0.38)$ \\
.0230 & 2.840 & 2.135 & 5.25 & $(0.54)$ \\
.0236 & 2.020 & 2.107 & 6.50 & $(0.66)$ \\
.0236 & 2.860 & 2.107 & 7.81 & $(0.79)$ \\
.0245 & 1.940 & 2.050 & 11.60 & $(1.18)$ \\
.0245 & 2.860 & 2.050 & 12.51 & $(1.27)$ \\
\hline
\end{tabular}

This manuscript reports the values of $g_{2}\left(r_{12} ; \rho, T\right)$ obtained from applying eq (1) to the data of Mozer, De Graaf, and Le Neindre; we also present the results of two other computations involving the pair function. The first is an investigation of an algorithm for the short range $\left(r_{12} \rightarrow 0\right)$ behavior of $g_{2}\left(r_{12} ; \rho, T\right)$. The other computation gives a comparison of the experimental results for the pair function and the results obtained from an approximate integral equation for the ground state value, $T=0$, of the pair function. 
Table 2. The pair correlation function, $g_{2}\left(r_{12} ; \rho, T\right)$, for liquid ${ }^{4}$ He as a function of internuclear distance, $\mathrm{r}_{12}$, temperature, $\mathrm{T}$, and density, $\rho$.

\begin{tabular}{|c|c|c|c|c|c|c|c|c|c|c|}
\hline $\begin{array}{l}\rho\left(\AA^{-3}\right) \\
T(K) \\
r_{12}(\AA)\end{array}$ & $\begin{array}{l}.0230 \\
1.860\end{array}$ & $\begin{array}{l}.0230 \\
2.050\end{array}$ & $\begin{array}{l}.0230 \\
2.130\end{array}$ & $\begin{array}{l}.0230 \\
2.140\end{array}$ & $\begin{array}{l}.0230 \\
2.300\end{array}$ & $\begin{array}{l}.0230 \\
2.840\end{array}$ & $\begin{array}{l}.0236 \\
2.020\end{array}$ & $\begin{array}{l}.0236 \\
2.860\end{array}$ & $\begin{array}{l}.0245 \\
1.940\end{array}$ & $\begin{array}{l}.0245 \\
2.860\end{array}$ \\
\hline .123 & .400 & .784 & 1.714 & 1.454 & 1.762 & 1.498 & 1.242 & .915 & .850 & .518 \\
\hline .368 & .481 & .681 & 1.329 & 1.228 & 1.389 & 1.254 & 1.088 & .929 & .780 & .638 \\
\hline .491 & .514 & .600 & 1.060 & 1.056 & 1.126 & 1.067 & .961 & .899 & .706 & .674 \\
\hline .614 & .518 & .509 & .789 & .865 & .857 & .857 & .810 & .823 & .606 & .658 \\
\hline 1.104 & .223 & .170 & .143 & .181 & .133 & .150 & .182 & .212 & .129 & .145 \\
\hline 1.227 & .127 & .111 & .089 & .071 & .051 & .059 & .075 & .080 & .049 & .025 \\
\hline 1.350 & .045 & .062 & .046 & -.011 & -.007 & .000 & -.001 & -.013 & -.007 & -.053 \\
\hline 1.473 & -.019 & .023 & .003 & -.068 & -.050 & -.035 & -.049 & -.069 & -.044 & -.090 \\
\hline 1.595 & -.064 & -.007 & -.045 & -.102 & -.083 & -.059 & -.075 & -.094 & -.066 & -.096 \\
\hline 1.718 & -.093 & -.027 & -.093 & -.118 & -.105 & -.079 & -.084 & -.099 & -.076 & -.082 \\
\hline 1.841 & -.108 & -.032 & -.128 & -.119 & -.115 & -.096 & -.079 & -.088 & -.072 & -.058 \\
\hline 2.086 & -.073 & .015 & -.107 & -.071 & -.075 & -.083 & -.013 & -.012 & .004 & .021 \\
\hline 2.209 & -.008 & .076 & -.033 & -010 & -.011 & -.027 & .060 & .062 & .086 & .091 \\
\hline 2.332 & .100 & .167 & .084 & .087 & .090 & .076 & .166 & .168 & .200 & .191 \\
\hline 2.454 & .252 & .289 & .241 & .226 & .233 & .228 & .307 & .307 & .344 & .327 \\
\hline 2.577 & .442 & .440 & .428 & .404 & .412 & .420 & .476 & .473 & .513 & .494 \\
\hline 2.700 & .654 & .615 & .632 & .614 & .619 & .636 & .663 & .659 & .698 & .684 \\
\hline 2.823 & .867 & .803 & .838 & .834 & .835 & .853 & .853 & .851 & .887 & .882 \\
\hline 2.945 & 1.060 & .990 & 1.031 & 1.043 & 1.039 & 1.053 & 1.032 & 1.035 & 1.066 & 1.071 \\
\hline 3.068 & 1.216 & 1.161 & 1.197 & 1.219 & 1.213 & 1.218 & 1.187 & 1.195 & 1.223 & 1.233 \\
\hline 3.191 & 1.326 & 1.299 & 1.325 & 1.345 & 1.341 & 1.339 & 1.308 & 1.320 & 1.343 & 1.355 \\
\hline 3.313 & 1.390 & 1.392 & 1.408 & 1.418 & 1.418 & 1.413 & 1.390 & 1.403 & 1.420 & 1.431 \\
\hline 3.436 & 1.415 & 1.435 & 1.448 & 1.444 & 1.448 & 1.444 & 1.430 & 1.440 & 1.451 & 1.460 \\
\hline 3.559 & 1.411 & 1.430 & 1.450 & 1.435 & 1.440 & 1.438 & 1.431 & 1.436 & 1.439 & 1.447 \\
\hline 3.682 & 1.387 & 1.388 & 1.421 & 1.404 & 1.407 & 1.405 & 1.398 & 1.399 & 1.394 & 1.403 \\
\hline 3.804 & 1.347 & 1.324 & 1.371 & 1.360 & 1.358 & 1.354 & 1.340 & 1.340 & 1.327 & 1.338 \\
\hline 3.927 & 1.295 & 1.251 & 1.307 & 1.307 & 1.301 & 1.293 & 1.267 & 1.269 & 1.251 & 1.263 \\
\hline 4.050 & 1.230 & 1.182 & 1.237 & 1.245 & 1.236 & 1.228 & 1.190 & 1.197 & 1.175 & 1.186 \\
\hline 4.172 & 1.156 & 1.122 & 1.164 & 1.174 & 1.167 & 1.161 & 1.118 & 1.129 & 1.104 & 1.112 \\
\hline 4.295 & 1.078 & 1.071 & 1.092 & 1.098 & 1.095 & 1.094 & 1.056 & 1.068 & 1.041 & 1.046 \\
\hline 4.418 & 1.003 & 1.028 & 1.024 & 1.021 & 1.024 & 1.029 & 1.006 & 1.014 & .988 & .987 \\
\hline 4.541 & .941 & .988 & .963 & .953 & .960 & .966 & .966 & .967 & .942 & .938 \\
\hline 4.663 & .898 & .949 & .911 & .900 & .908 & .910 & .932 & .925 & .904 & .897 \\
\hline 4.786 & .872 & .911 & .871 & .865 & .870 & .866 & .901 & .889 & .875 & .865 \\
\hline 4.909 & .861 & .879 & .843 & .846 & .846 & .837 & .874 & .862 & .853 & .842 \\
\hline 5.031 & .859 & .856 & .827 & .838 & .836 & .827 & .852 & .844 & .840 & .829 \\
\hline 5.154 & .860 & .845 & .823 & .836 & .834 & .831 & .840 & .836 & .836 & .827 \\
\hline 5.277 & .861 & .846 & .830 & .837 & .830 & .847 & .839 & .840 & .842 & .834 \\
\hline 5.400 & .866 & .858 & .847 & .845 & .850 & .867 & .851 & .852 & .857 & .852 \\
\hline 5.522 & .876 & .879 & .871 & . 860 & .867 & - 886 & .874 & .873 & .880 & .878 \\
\hline 5.645 & .895 & .905 & .898 & .885 & .890 & .904 & .904 & .899 & .911 & .910 \\
\hline 5.768 & .923 & .934 & .926 & .919 & .919 & .923 & .935 & .929 & .944 & .944 \\
\hline 5.890 & .955 & .962 & .953 & .957 & .952 & .943 & .965 & .059 & .078 & .978 \\
\hline 6.013 & .987 & .987 & .979 & .992 & .984 & .969 & .991 & .988 & 1.007 & 1.008 \\
\hline 6.136 & 1.015 & 1.009 & 1.005 & 1.021 & 1.013 & .999 & 1.013 & 1.014 & 1.030 & 1.032 \\
\hline 6.259 & 1.035 & 1.027 & 1.030 & 1.040 & 1.038 & 1.030 & 1.031 & 1.035 & 1.046 & 1.050 \\
\hline 6.381 & 1.049 & 1.039 & 1.056 & 1.053 & 1.057 & 1.058 & 1.046 & 1.052 & 1.057 & 1.064 \\
\hline 6.504 & 1.059 & 1.048 & 1.078 & 1.061 & 1.072 & 1.078 & 1.058 & 1.065 & 1.065 & 1.074 \\
\hline 6.627 & 1.067 & 1.054 & 1.094 & 1.070 & 1.082 & 1.088 & 1.068 & 1.073 & 1.071 & 1.080 \\
\hline 6.750 & 1.073 & 1.059 & 1.099 & 1.079 & 1.088 & 1.089 & 1.073 & 1.077 & 1.075 & 1.083 \\
\hline 6.872 & 1.076 & 1.062 & 1.094 & 1.086 & 1.087 & 1.085 & 1.074 & 1.077 & 1.075 & 1.081 \\
\hline 6.995 & 1.073 & 1.064 & 1.078 & 1.087 & 1.080 & 1.076 & 1.069 & 1.073 & 1.071 & 1.073 \\
\hline
\end{tabular}

We proceed next, to the results for $g_{2}\left(r_{12} ; \rho, T\right)$ and then in section 3 , we discuss the short range behavior. The computation of the pair function from the PercusYevick approximation will be given in section 4 ; the article concludes, at section 5 , with a discussion of results.

\section{Pair Function from Scattering Data}

The results of applying eq (1) to the neutron diffraction measurements are given in table 2 , where we have listed $g_{2}\left(r_{12} ; \rho, T\right)$ as a function of $r_{12}$ for all the thermodynamic states shown in table 1 . 
Table 2, continued. The pair correlation function, $g_{2}\left(r_{12} ; \rho, T\right)$, for liquid ${ }^{4}$ He as a function of internuclear distance, $\mathrm{r}_{12}$, temperature, $\mathrm{T}$, and density, $\rho$.

\begin{tabular}{|c|c|c|c|c|c|c|c|c|c|c|}
\hline 7.118 & 1.063 & 1.062 & 1.058 & 1.079 & 1.067 & 1.066 & 1.061 & 1.065 & 1.062 & 1.061 \\
\hline 7.240 & 1.047 & 1.055 & 1.038 & 1.062 & 1.049 & 1.053 & 1.049 & 1.053 & 1.047 & 1.045 \\
\hline 7.363 & 1.028 & 1.042 & 1.022 & 1.039 & 1.030 & 1.038 & 1.034 & 1.037 & 1.028 & 1.027 \\
\hline 7.486 & 1.009 & 1.025 & 1.011 & 1.016 & 1.012 & 1.021 & 1.019 & 1.020 & 1.009 & 1.008 \\
\hline 7.600 & .095 & 1.007 & 1.003 & .995 & .998 & 1.003 & 1.003 & 1.003 & . 991 & .991 \\
\hline 7.731 & .986 & .991 & .996 & . 982 & .988 & .987 & .990 & .988 & .977 & .977 \\
\hline 7.854 & .981 & .979 & .986 & .974 & .980 & .975 & .978 & .976 & .968 & .966 \\
\hline 7.977 & .979 & .972 & .974 & .970 & .973 & .967 & .071 & .068 & .063 & .959 \\
\hline 8.099 & .975 & .970 & .963 & .968 & .966 & .963 & .966 & .063 & .961 & .956 \\
\hline 8.222 & .971 & .971 & .955 & .965 & .960 & .962 & .064 & .062 & .961 & .956 \\
\hline 8.345 & .967 &.$\quad .972$ & .952 & .962 & .956 & .960 & .965 & .962 & .963 & .958 \\
\hline 8.468 & .965 & .973 & .955 & .959 & .955 & .959 & .967 & .963 & .965 & .963 \\
\hline 8.590 & .965 & .973 & .962 & . 958 & . 959 & .959 & .969 & .965 & .969 & .968 \\
\hline 8.713 & .970 & .973 & .970 & .961 & .966 & .961 & .972 & .969 & .974 & .974 \\
\hline 8.836 & .977 & .976 & .977 & .968 & .976 & .968 & .976 & .974 & .980 & .980 \\
\hline 8.958 & .085 & .081 & .983 & .977 & .985 & .978 & .980 & .981 & .987 & .987 \\
\hline 9.081 & .993 & .987 & .989 & . 988 & .992 & .991 & .987 & .988 & .994 & . 994 \\
\hline 9.204 & 1.000 & .995 & .997 & .999 & .999 & 1.003 & .994 & .097 & 1.000 & 1.002 \\
\hline 9.327 & 1.005 & 1.002 & 1.006 & 1.008 & 1.005 & 1.012 & 1.002 & 1.005 & 1.007 & 1.011 \\
\hline 9.449 & 1.010 & 1.007 & 1.015 & 1.016 & 1.010 & 1.018 & 1.010 & 1.011 & 1.013 & 1.018 \\
\hline 9.572 & 1.014 & 1.011 & 1.021 & 1.022 & 1.017 & 1.020 & 1.017 & 1.017 & 1.019 & 1.024 \\
\hline 9.695 & 1.018 & 1.014 & 1.025 & 1.026 & 1.023 & 1.021 & 1.022 & 1.022 & 1.023 & 1.028 \\
\hline 9.817 & 1.021 & 1.016 & 1.024 & 1.027 & 1.027 & 1.022 & 1.025 & 1.025 & 1.026 & 1.029 \\
\hline 9.940 & 1.022 & 1.019 & 1.021 & 1.026 & 1.029 & 1.022 & 1.025 & 1.026 & 1.027 & 1.027 \\
\hline 10.063 & 1.021 & 1.020 & 1.019 & 1.024 & 1.027 & 1.022 & 1.023 & 1.026 & 1.025 & 1.023 \\
\hline 10.186 & 1.018 & 1.019 & 1.018 & 1.021 & 1.022 & 1.021 & 1.019 & 1.023 & 1.021 & 1.018 \\
\hline 10.308 & 1.014 & 1.015 & 1.017 & 1.017 & 1.016 & 1.018 & 1.014 & 1.018 & 1.015 & 1.012 \\
\hline 10.431 & 1.009 & 1.011 & 1.017 & 1.012 & 1.010 & 1.014 & 1.009 & 1.011 & 1.008 & 1.007 \\
\hline 10.554 & 1.004 & 1.006 & 1.014 & 1.007 & 1.005 & 1.008 & 1.004 & 1.005 & 1.002 & 1.001 \\
\hline 10.677 & 1.000 & 1.002 & 1.009 & 1.002 & 1.001 & 1.003 & 1.000 & .999 & .997 & .997 \\
\hline 10.799 & .997 & 1.000 & 1.001 & .998 & .999 & .998 & .998 & .996 & .994 & - 994 \\
\hline 10.922 & .094 & .999 & .993 & - 994 & .997 & .393 & .996 & .995 & .992 & .992 \\
\hline 11.045 & .992 & .999 & .987 & .991 & .994 & .989 & .995 & .994 & .991 & .990 \\
\hline 11.167 & .991 & - 998 & .985 & .990 & . 991 & .986 & .993 & .093 & .991 & .989 \\
\hline 11.290 & .990 & .995 & .985 & .989 & .987 & .985 & .991 & .991 & .990 & .987 \\
\hline 11.413 & .989 & .991 & .987 & . 988 & .984 & .984 & .989 & .988 & .988 & .985 \\
\hline 11.536 & .989 & .988 & .988 & .986 & .982 & .985 & .988 & .985 & .987 & . 984 \\
\hline 11.658 & .990 & - 086 & .989 & .985 & .983 & .988 & .987 & . 984 & .986 & - 984 \\
\hline 11.781 & .992 & .987 & .988 & .985 & .986 & .992 & .088 & .985 & .987 & .987 \\
\hline 11.904 & .995 & .990 & .988 & .986 & .991 & .905 & .091 & .088 & .989 & .992 \\
\hline 12.026 & .998 & .995 & .990 & .990 & .996 & - 99,8 & .994 & .993 & .994 & .997 \\
\hline 12.149 & 1.000 & .999 & .993 & . 994 & 1.001 & 1.000 & .097 & .099 & .999 & 1.002 \\
\hline 12.272 & 1.002 & 1.001 & .997 & .999 & 1.003 & 1.001 & 1.001 & 1.003 & 1.003 & 1.006 \\
\hline 12.395 & 1.002 & 1.002 & 1.002 & 1.003 & 1.004 & 1.002 & 1.003 & 1.005 & 1.007 & 1.007 \\
\hline 12.517 & 1.002 & 1.001 & 1.005 & 1.006 & 1.003 & 1.003 & 1.004 & 1.005 & 1.008 & 1.007 \\
\hline 12.640 & 1.001 & 1.001 & 1.007 & 1.007 & 1.003 & 1.005 & 1.005 & 1.005 & 1.008 & 1.006 \\
\hline 12.763 & 1.001 & 1.002 & 1.008 & 1.007 & 1.003 & 1.006 & 1.005 & 1.006 & 1.007 & 1.006 \\
\hline 12.885 & 1.002 & 1.003 & 1.009 & 1.007 & 1.004 & 1.006 & 1.005 & 1.007 & 1.006 & 1.007 \\
\hline 13.008 & 1.004 & 1.005 & 1.009 & 1.008 & 1.006 & 1.006 & 1.005 & 1.008 & 1.006 & 1.008 \\
\hline 13.131 & 1.005 & 1.006 & 1.009 & 1.009 & 1.008 & 1.005 & 1.005 & 1.009 & 1.007 & 1.009 \\
\hline 13.254 & 1.006 & 1.007 & 1.008 & 1.009 & 1.009 & 1.004 & 1.005 & 1.009 & 1.007 & 1.009 \\
\hline 13.376 & .1 .005 & 1.006 & 1.006 & 1.009 & 1.009 & 1.004 & 1.005 & 1.007 & 1.006 & 1.007 \\
\hline 13.499 & 1.004 & 1.004 & 1.004 & 1.007 & 1.006 & 1.003 & 1.004 & 1.005 & 1.004 & 1.004 \\
\hline 13.622 & 1.002 & 1.003 & 1.002 & 1.005 & 1.004 & 1.002 & 1.003 & 1.002 & 1.002 & 1.000 \\
\hline 13.744 & 1.001 & 1.002 & 1.001 & 1.002 & 1.001 & 1.001 & 1.003 & .999 & 1.000 & . 998 \\
\hline 13.867 & 1.000 & 1.001 & 1.000 & 1.000 & .999 & 1.000 & 1.002 & .097 & .998 & . 997 \\
\hline 13.990 & 1.001 & 1.001 & 1.000 & .998 & . 998 & .998 & 1.001 & .996 & .997 & . 996 \\
\hline 14.113 & 1.001 & .1 .000 & .999 & .997 & . 998 & .997 & .999 & .996 & .997 & .996 \\
\hline 14.235 & 1.000 & .999 & .997 & .995 & .997 & .996 & .998 & .996 & .997 & .996 \\
\hline 14.358 & .998 & .997 & .995 & .994 & .997 & .997 & .096 & .096 & .997 & .996 \\
\hline 14.481 & .996 & .996 & .993 & .992 & . 996 & .997 & .995 & .996 & .997 & .995 \\
\hline
\end{tabular}

There are two regions of $r_{12}$ which reflect the principal uncertainties in measurements of $S(\kappa ; \rho, T)$; these are the small and large $r_{12}$ values. The structure factor is positive and must satisfy,

$$
\lim _{\kappa \rightarrow 0} S(\kappa ; \rho, T)=\rho k_{B} T \kappa_{T}
$$

and

$$
\lim _{\kappa \rightarrow \infty} S(\kappa ; \rho, T)=1
$$

where $k_{B}$ is the Boltzmann constant and $\kappa_{T}$ the isothermal compressibility. The experimentally accessible regions of $\kappa$, the magnitude of the change in the neutron wave vector upon scattering, is limited. Therefore, in practice, one must necessarily extrapolate from a given small value of $\kappa$ to zero in order to satisfy eq $(2)$ and one must also truncate $S(\kappa ; \rho, T)$ at a finite value of $\kappa$ in satisfying eq (3). In the Fourier transform, the errors introduced in satisfying eq (2) 
Table 2, continued. The pair correlation function, $g_{2}\left(r_{12} ; \rho, T\right)$, for liquid ${ }^{4}$ He as a function of internuclear distance, $\mathrm{r}_{12}$, temperature, $\mathrm{T}$, and density, $\rho$.

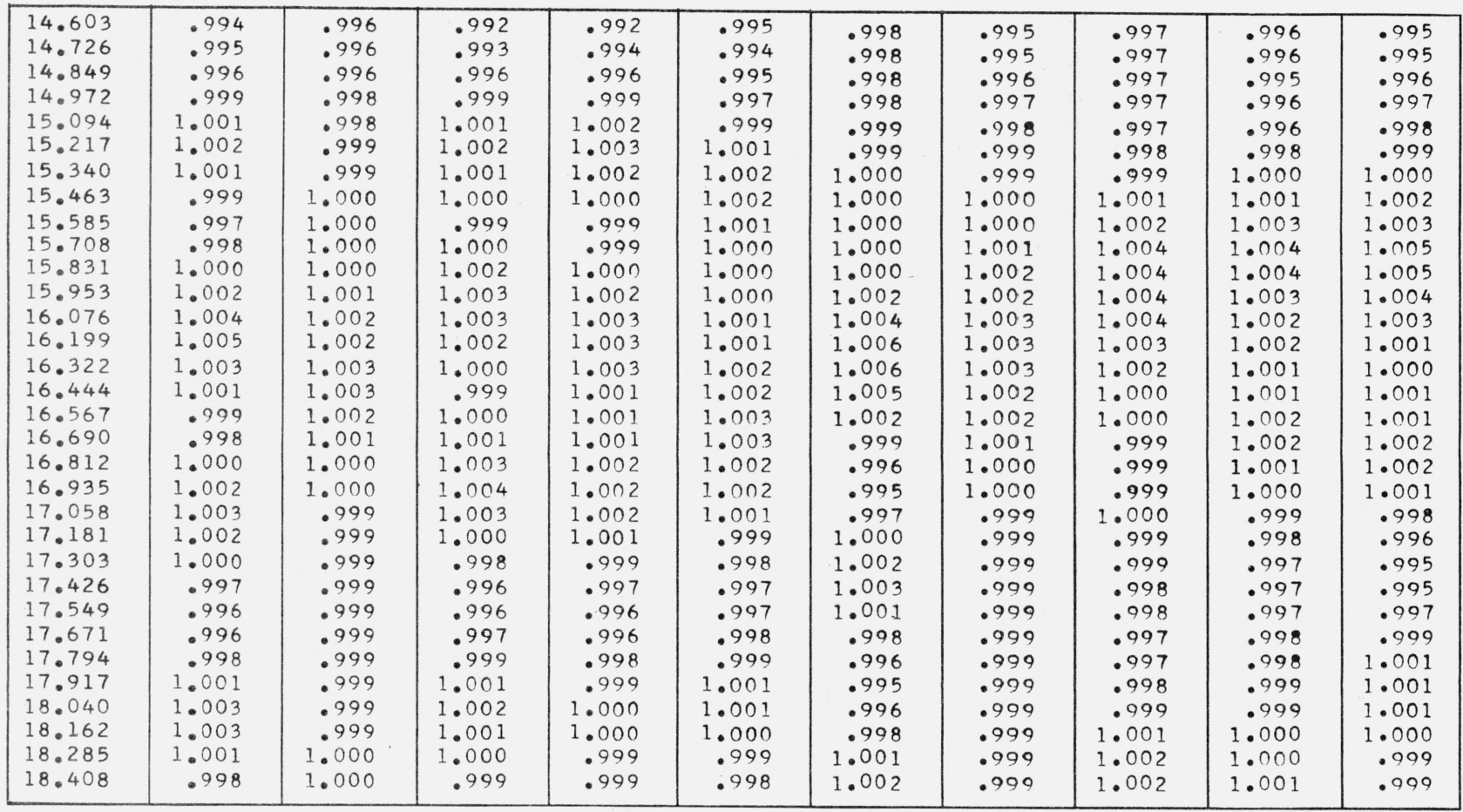

effect the accuracy of the large $\mathrm{r}_{12}$ behavior of $g_{2}\left(r_{12} ; \rho, T\right)$. The errors introduced in satisfying eq (3) give the unphysical oscillations about the value zero which are shown in table 2. Mozer et al. have given $[6,7]$ an extended discussion of the extrapolation to $\kappa=0$, and we will, in the following section, discuss an algorithm to smoothly and accurately extend $g_{2}\left(r_{12} ; \rho, T\right)$ to the value zero which it must necessarily have at $r_{12}=0$. It suffices to mention here that in the numerical evaluation of the Fourier transform, eq (1), $S(\kappa ; \rho, T)$ was truncated to unity for $\kappa \geqslant 7 \AA^{-1}$. The statistical accuracy of the resulting pair functions is estimated to be within \pm 3 percent.

\section{Short Range Behavior}

The negative values of $g_{2}\left(r_{12} ; \rho, T\right)$ in table 2 are unphysical and are introduced into eq (1) by truncating $S(\kappa ; \rho, T)$. For physical systems, the pair function must approach zero in value as the internuclear distance approaches zero, and the approach to zero is presumably monotone.

We consider a method for smoothly extrapolating the results in table 2 to the value $g_{2}=0$; as we will discuss, the method also applies to classical liquids. Consider the pair function for $r_{12} \leqslant d$ and denote the function on this interval by $\tilde{\mathrm{g}}_{2}\left(r_{12} \leqslant d\right)$. The form $[8,9]$

$\tilde{\mathrm{g}}_{2}\left(r_{12} \leqslant d\right)=g_{2}(d ; \rho, T) \exp \left\{\lambda\left[1-\left(\frac{d}{r_{12}}\right)^{m}\right]\right\}$ will be investigated, where we choose $d$ as the smallest value of $r_{12}$ for which the pair function has a statistical accuracy equivalent to that at larger $r_{12}$ values. The number $g_{2}(d ; \rho, T)$ is the value of $g_{2}\left(r_{12} ; \rho, T\right)$ at $r_{12}=d$. For a given value of $m$, the quantity $\lambda$ is determined by the continuity of the derivative of the pair function and $\tilde{\mathrm{g}}_{2}\left(r_{12} \leqslant d\right)$,

$$
\lambda=\frac{d}{m}\left(\frac{g_{2}^{\prime}\left(r_{12} ; \rho, T\right)}{g_{2}\left(r_{12} ; \rho, T\right)}\right)_{r_{12}=d} .
$$

The values $m=5$ and $m=10$ were considered and the typical agreement is shown in figures 1 and 2 . In figure 1, the solid curve is the experimental result for ${ }^{4} \mathrm{He}$ at $\rho=0.0230 / \AA^{3}$ and $T=2.050 \mathrm{~K}$; the crisscross denotes the value of $d$. The dashed curve is for $m=5$ and $\lambda=2.423$, and the dashed-dotted curve for $m=10$ and $\lambda=1.211$. Similarly in figure 2 , the solid curve is the experimental result for ${ }^{4} \mathrm{He}$ at $\rho=0.0230 / \AA^{3}$ and $T=2.840 \mathrm{~K}$; the dashed curve is eq (2) with $m=5$, $\lambda=2.032$ and the dashed-dotted curve corresponds to $m=10, \lambda=1.016$. As we have already mentioned, the results in figures 1 and 2 are typical of the results obtained using all the helium data in table 2 , and from this we infer $m=5$ is a good choice for ${ }^{4} \mathrm{He}$. To determine whether the algorithm also has utility in classical liquids, we investigated the data of Yarnell, Katz, Wenzel, and Koenig [10] taken for liquid argon near its triple point, $\rho=0.0215 / \AA^{3}$ and $T=85 \mathrm{~K}$. The results are shown in figure 3 where the solid curve is the experimental data, the dashed-dotted curve is eq (2) with $m=12, \lambda=2.670$ and the dashed curve corresponds 


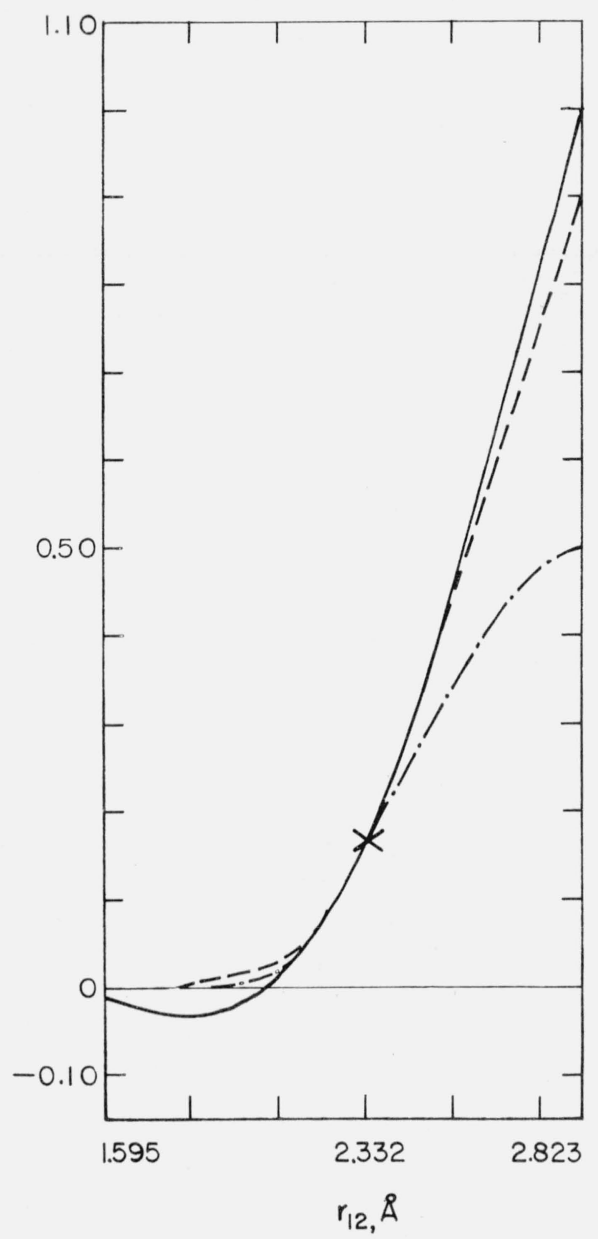

FIGURE 1. The short range behavior of $\mathrm{g}_{2}\left(\mathrm{r}_{12}\right)$ in liquid ${ }^{4} \mathrm{He}$.

The solid curve is the experimental result at $\rho=0.0230 / \AA^{3}, T=2.050 \mathrm{~K}$; the dashed curve is $\tilde{g}\left(r_{12} \leqslant d\right)$, eq (4), with $m=5, \lambda=2.423$; the dashed-dotted curve is the same function with $m=10, \lambda=1.211$. The crisscross denotes the value of $d$.

to $m=5, \lambda=6.407$. Relative to the data for ${ }^{4} \mathrm{He}$, the argon results suggest that for classical fluids, $m=12$ is a better choice.

\section{Percus-Yevick Approximation}

For a fluid system at $T=0$, where the wave function, $\Psi_{N}^{(0)}$, for a set of $N$ atoms is given by the Bijl-DingleJastrow form [11],

$$
\Psi_{N}^{(0)}=\prod_{1 \leqslant i<j \leqslant N} \exp \left\{-\frac{1}{2} \phi\left(r_{i j}\right)\right\},
$$

the Percus-Yevick (P.Y.) approximation [12], may be used [13] to obtain an approximate integral equation for $g_{2}\left(r_{12}\right)$ in terms of $\phi\left(r_{12}\right)$. The result is,

$g_{2}\left(r_{12}\right)=\exp \left\{-\phi\left(r_{12}\right)\right\}\left[1+\rho \int d \mathbf{r}_{3} g_{2}\left(r_{13}\right)\left[g_{2}\left(r_{23}\right)-1\right]\right.$

$$
\left.\left[1-\exp \left\{\phi\left(r_{13}\right)\right\}\right]\right] \text {. }
$$

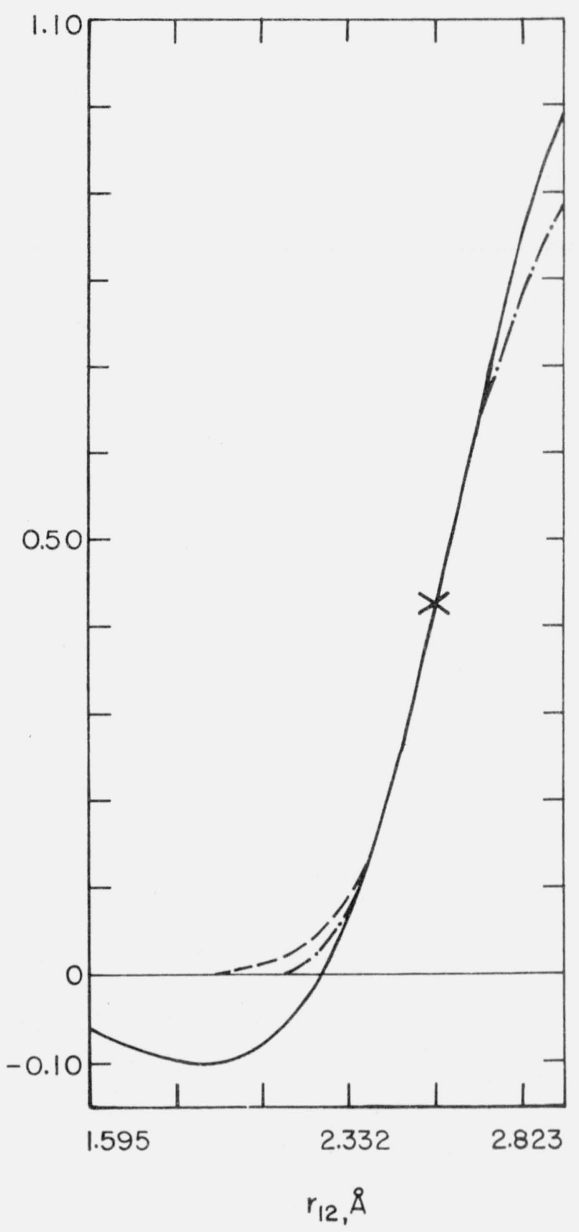

FIgURE 2. The same as figure 1, except the experimental result is at $\mathrm{T}=2.840 \mathrm{~K}$; the dashed curve is for $\mathrm{m}=5, \lambda=2.032$; the dasheddotted curve for $\mathrm{m}=10, \lambda=1.016$.

For $2.332 \AA \leqslant r_{12} \leqslant 2.867$, the dashed curve is identical to the solid curve.

Shiff and Verlet [14] have found from computer simulation studies, that for a Hamiltonian whose interaction energy, $U\left(r_{12}\right)$, is the Lennard-Jones function,

$$
U\left(r_{12}\right) \sim\left[\left(\frac{\sigma}{r_{12}}\right)^{12}-\left(\frac{\sigma}{r_{12}}\right)^{6}\right],
$$

the function

$$
\phi\left(r_{12}\right)=\left(\frac{1.16 \sigma}{r_{12}}\right)^{5}
$$

minimizes the ground state energy. Using eq (8) and the algorithm given by Mandel, Bearman, and Bearman [15], we have solved eq (7) for $g_{2}\left(r_{12}\right)$. All computations were done in units of $\sigma$, which for ${ }^{4} \mathrm{He}$ is [14],

$$
\sigma=2.556 \AA \text {. }
$$




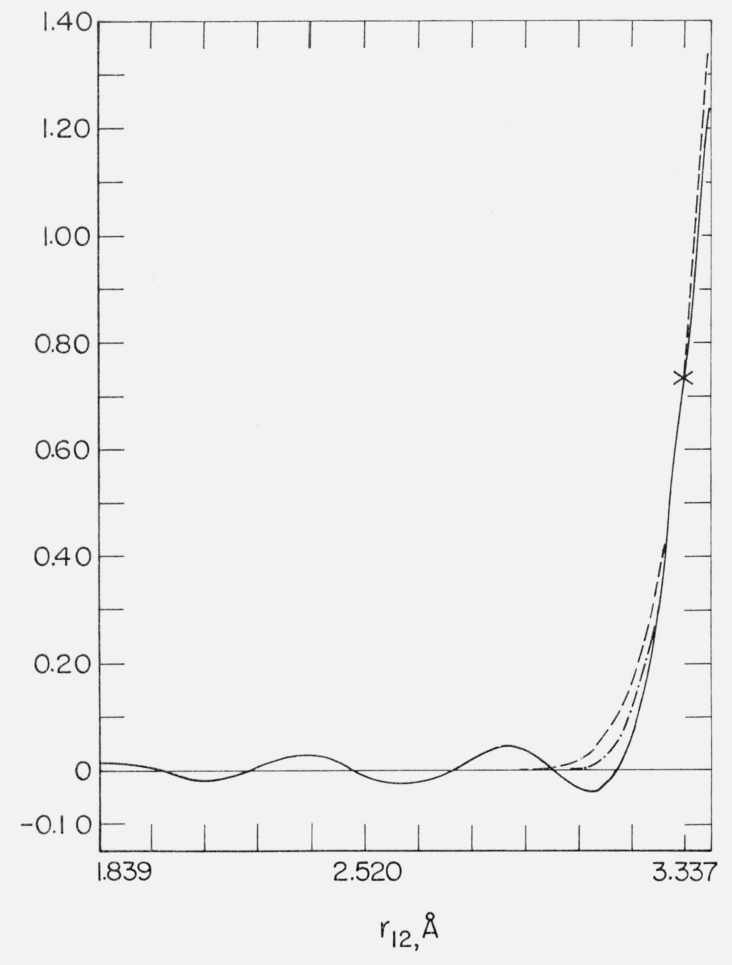

Figure 3. The short range behavior of $\mathrm{g}_{2}\left(\mathrm{r}_{12}\right)$ in liquid Ar.

The solid curve is the experimental resuit from reference $[8] ; \rho=0.0215 / \AA^{3}, T=85 \mathrm{~K}$. The dashed curve is eq (4) with $m=5, \lambda=6.407$; the dashed-dotted curve is the same function with $m=12, \lambda=2.700$. The crisscross denotes the value of $d$. For the largest $r_{12}$ values shown, the dashed-dotted curve is identical to the solid curve.

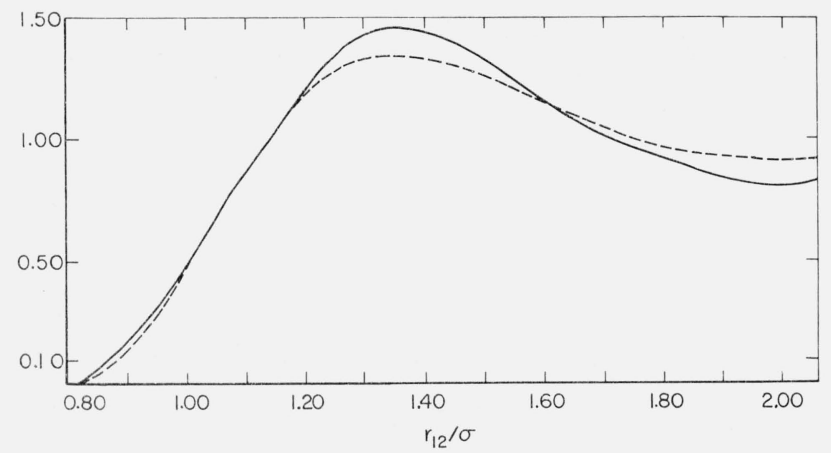

Figure 4. The pair correlation function for liquid ${ }^{4} \mathrm{He}$ in units of $\sigma=2.556 \AA$ at $\rho \sigma^{3}=0.409$.

The solid curve is the experimental result at $T=1.940 \mathrm{~K}$. The dashed curve is the P.Y. approximation, eq (7), with the ground state function defined by eqs (6) and (8).

The results for $\rho \sigma^{3}=0,409$, which with eq (9) corresponds to $\rho=0.0245 / \AA^{3}$, are shown in figure 4 . The dashed curve is the P.Y. solution and the solid curve is the measured value at $\rho=0.0245 / \AA^{3}$ and $T=1.940 \mathrm{~K}$. In considering the relative agreement, which is within \pm 15 percent, we wish to emphasize that there are three sources of error: (a) the uncertainty between eqs (6) and (8) and the true ground state of helium, (b) the P.Y. approximation, (c) the contribution of excited

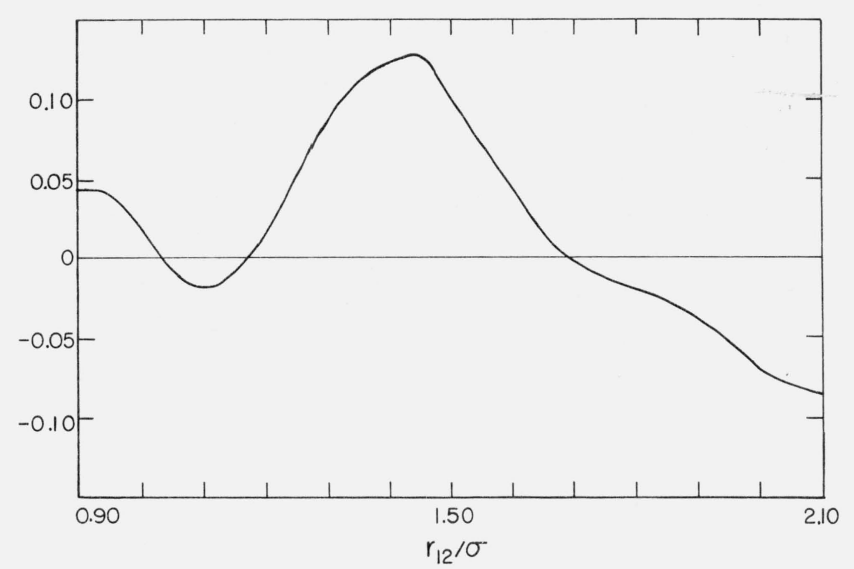

FIGURE 5. The difference, $\mathrm{g}_{2}^{\text {expt. }}-\mathrm{g}_{2}^{P . Y}$, at $\rho \sigma^{3}=0.394$.

The experimental result is at $T=2.020 \mathrm{~K}$.

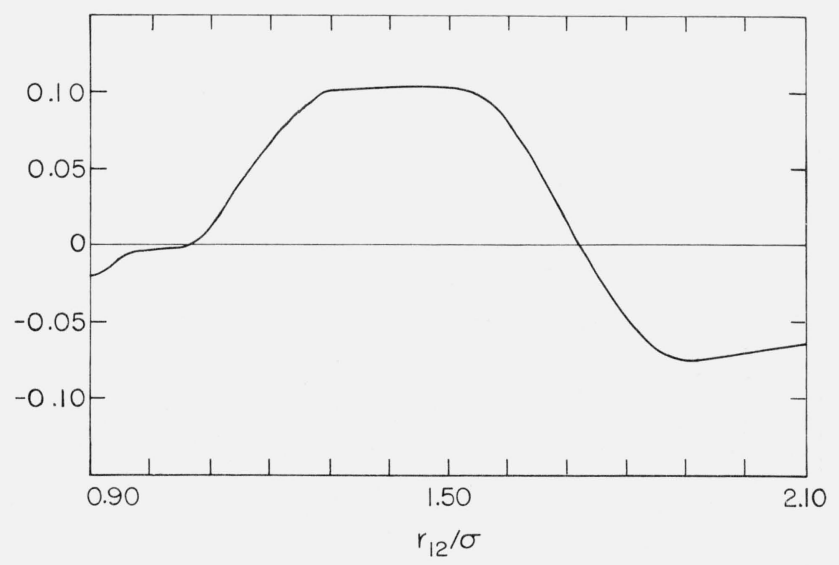

Figure 6. Same as figure 5, except $\rho \sigma^{3}=0.394$ and experimental result is at $\mathrm{T}=1.860 \mathrm{~K}$.

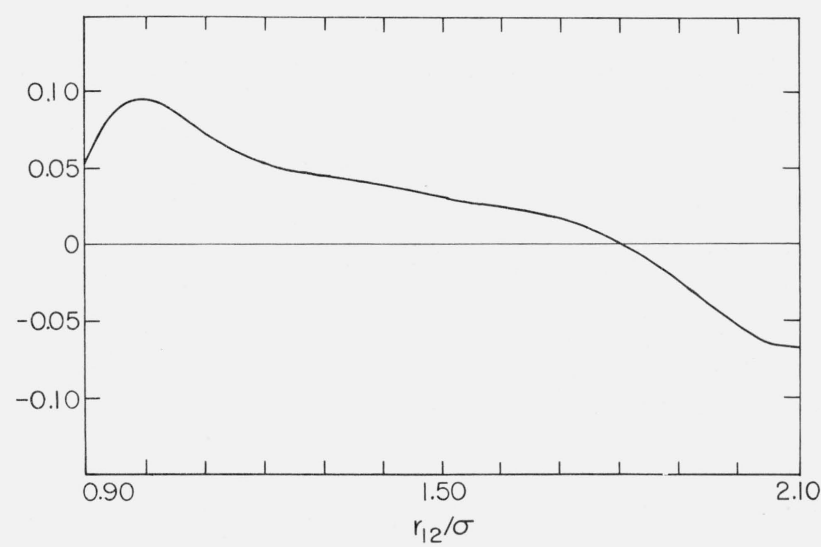

Figure 7. Same as figure 5, except $\rho \sigma^{3}=0.365$ and experimental result (ref. [14]) is at $\mathrm{T}=0.79 \mathrm{~K}$. 
quantum states. Keeping this in mind, it is of interest to consider effects of finite temperature. The results arc shown in figures 5,6 , and 7 , where we have plotted $g_{2}^{\text {expt. }}-g_{2}^{\text {P.Y. }}$ for $\rho \sigma^{3}=0.394,0.384$, and 0.365 respectively. The experimental data used in constructing figures 5 and 6 correspond, respectively, to the measurements at $\rho=0.0236 / \AA^{3}, T=2.020 \mathrm{~K}$ and $\rho=0.0230 / \AA^{3}$, $T=1.860 \mathrm{~K}$. The experimental data used in figure 7 were obtained from the x-ray measurements of Achter and Meyer [3]; these data are at $\rho=0.0219 / \AA^{3}\left(\rho \sigma^{3}\right.$ $=0.365$ ) and $T=0.79 \mathrm{~K}$. The results in figures 5,6 , and 7 indicate that at intermediate values of the internuclear separation, the P.Y. approximation characteristically underestimates the pair correlation in liquid ${ }^{4} \mathrm{He}$, and at larger values of $r_{12} / \sigma$, the P.Y. approximation characteristically overestimates the measured values. Little, if anything, can be inferred from the differences at small internuclear separations, $r_{12} / \sigma<1$, since the measurements are, as we have already remarked, not as accurate as the data at larger separations.

\section{Discussion}

In a separate article [16], we have employed the data in table 2 in the study of several properties of the pair and triplet correlation functions. We reiterate one property that is particularly germane to this article. We consider table 2 in the vicinity of the first maximum of $g_{2}\left(r_{12} ; \rho, T\right), r_{12} \sim 3.5 \AA$, and the second minimum, $r_{12} \sim 5.3 \AA$. The magnitude of $g_{2}\left(r_{12} ; \rho, T\right)-1$ is referred to as the structure in the pair function. Table 2 shows that below $T_{\lambda}$, the structure is a decreasing function of temperature as the temperature is lowered at fixed density. The same dependence on temperature is observed as the temperature is lowered, at fixed density, from above $T_{\lambda}$, but below the boiling point, to temperatures below the superfluid transition. For classical fluids the pair correlation function is [16] an increasing function of temperature as the temperature is lowered at fixed density.

The algorithm given in eq (4) is, from figures 1,2 , and 3 , a simple and accurate procedure for smoothly extending the values of the pair function inside the first maximum, to the value $g_{2}=0$. The short range behavior is very difficult to measure and perhaps the algorithm will prove useful in presenting experimental results from diffraction experiments. As we have already noted, the method appears to have utility in classical as well as quantum fluids.

It should be emphasized that the computations given in section 4 strictly apply to a fluid whose wave function at the absolute zero of temperature is given by eq (6). With this in mind, the results in figures 4 thru 7 show the P.Y. approximation is with \pm 15 percent of the experimental results. The P.Y. approximation typically underestimates the magnitude and extent of the first peak in $g_{2}\left(r_{12}\right)$ but overestimates the function at somewhat larger $\left(r_{12} \geqslant 1.8 \sigma\right)$ values of the internuclear separation. As expected, the agreement between the calculated and experimental results improves when the calculated results are compared to data at lower temperatures.

The authors express their appreciation to B. Mozer for the use of the diffraction data prior to publication.

\section{References}

[1] Larsson, K., Dahlborg, U., and Sköld, K., in Simple Dense Fluids, edited by H. L. Frisch and Z. W. Salsburg (Academic Press, New York, 1968).

[2] Ibid.

[3] Achter, E. K., and Meyer, L., Phys. Rev. 188, 291 (1969).

[4] London, F., Superfluids, Vol. II, (John Wiley \& Sons, New York, 1954).

[5] Raverché, H. J., and Mountain, R. D., J. Chem. Phys. 53, 3107 (1970).

[6] Mozer, B., Le Neindre, B., and De Graaf, L. A., Neutron Diffraction Studies in Liquid ${ }^{4} \mathrm{He}$, submitted to Phys. Rev. A.

[7] Mozer, B., and Le Neindre, B., Neutron diffraction study of liquid ${ }^{4} \mathrm{He}$ at constant density, submitted to Phys. Rev. A.

[8] Wu, F. Y., and Feenberg, E., Phys. Rev. 122, 739 (1961).

[9] Woo, C. W., and Massey, W. E., Phys. Rev. 164, 256 (1967).

[10] Yarnell, J. L., Katz, M. J., Wenzel, R. G., and Koenig, S. H., Phys. Rev. A7, 2130 (1973).

[11] Bijl, A., Physica 7, 869 (1940); Dingel, R. B., Phil. Mag. 40, 573 (1949); Jastrow, R., Phys. Rev. 98, 1479 (1955).

[12] Percus, J. K., and Yevick, G. Y., Phys. Rev. 110,1 (1958).

[13] Feenberg, E., Theory of Quantum Fluids (Academic Press, New York, 1969), Chapter 6.

[14] Schiff, D., and Verlet, L., Phys. Rev. 160, 208 (1967).

[15] Mandel, F., Bearman, R. J., and Bearman, M. Y., J. Chem. Phys. 52, 3315 (1970).

[16] Raveché, H. J., and Mountain, R. D., Structure studies in liquid ${ }^{4} \mathrm{He}$, submitted to Phys. Rev. A.

(Paper 77A6-795) 